\title{
The perception of work stressors is related to reduced parasympathetic activity
}

\author{
Els Clays ${ }^{1}$, Dirk De Bacquer ${ }^{1}$, Vincent Crasset $^{2}$, France Kittel ${ }^{2}$, \\ Patrick de Smet ${ }^{2}$, Marcel Kornitzer ${ }^{2}$, Robert Karasek ${ }^{3}$, Guy De Backer ${ }^{1}$ \\ ${ }^{1}$ Department of Public Health, Ghent University, University Hospital, Block A, De Pintelaan \\ 185, 9000 Ghent, Belgium \\ 2 Department of Epidemiology and Health Promotion, School of Public Health, Free \\ University of Brussels, Brussels, Belgium \\ 3 Department of Work Environment, University of Massachusetts, Lowell, USA and \\ Department of Psychology, Copenhagen University, Copenhagen, Denmark
}

Background. The autonomic nervous system has recently been suggested as a possible physio-pathological pathway in the relation between stress and coronary heart disease. The aim was to examine work stressors in relation to measures of heart rate variability (HRV).

Methods. Results are based on observations in a sample of 653 healthy males aged 40-55 years from the Belgian Physical Fitness Study (1976-'78). Data were collected by means of questionnaires and bio-clinical examinations. An index of work stressors was constructed based on 5 items dealing with job satisfaction, physical working conditions, responsibility, work rhythm and social relations. Data on HRV were collected by means of 24-hour ambulatory ECG recordings on a working day. Both time and frequency domain measures of HRV were calculated.

Results. The Work Stressor Index was significantly associated with lower pNN50 (\% of differences between adjacent normal RR intervals $>50 \mathrm{~ms}$ ), lower high frequency power and a higher ratio of low frequency over high frequency power. Similar results were obtained after adjusting for age, language, occupation, smoking, body mass index, total cholesterol, systolic blood pressure, and leisure time physical activity. No significant associations were found with SDNN (the SD of all normal RR intervals) and low frequency power.

Conclusion. The perception of work stressors was related to reduced HRV in a sample of 653 healthy males. These results suggest that disturbances of the autonomic nervous system and its parasympathetic component in particular may play a role in the link between work stress and coronary heart disease. 